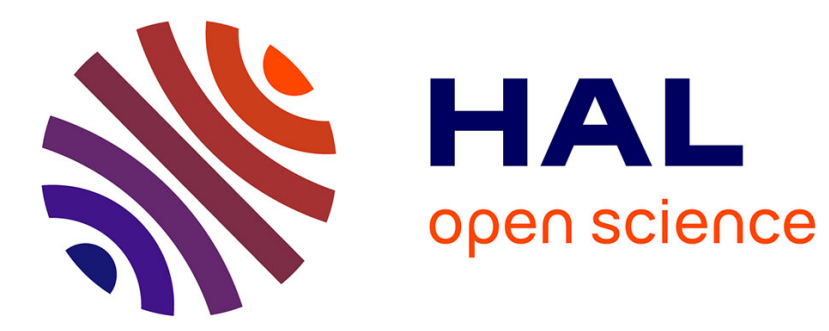

\title{
Combining Formation Seismic Velocities while Drilling and a PDE-ODE observer to improve the Drill-String Dynamics Estimation
}

\author{
Jean Auriol, Nasser Kazemi, Kristopher Innanen, Roman Shor
}

\section{To cite this version:}

Jean Auriol, Nasser Kazemi, Kristopher Innanen, Roman Shor. Combining Formation Seismic Velocities while Drilling and a PDE-ODE observer to improve the Drill-String Dynamics Estimation. ACC 2020 - American Control Conference, Jul 2020, Denver, United States. hal-02524281

\section{HAL Id: hal-02524281 \\ https://hal.science/hal-02524281}

Submitted on 30 Mar 2020

HAL is a multi-disciplinary open access archive for the deposit and dissemination of scientific research documents, whether they are published or not. The documents may come from teaching and research institutions in France or abroad, or from public or private research centers.
L'archive ouverte pluridisciplinaire HAL, est destinée au dépôt et à la diffusion de documents scientifiques de niveau recherche, publiés ou non, émanant des établissements d'enseignement et de recherche français ou étrangers, des laboratoires publics ou privés. 


\title{
Combining Formation Seismic Velocities while Drilling and a PDE-ODE observer to improve the Drill-String Dynamics Estimation
}

\author{
Jean Auriol ${ }^{1}$, Nasser Kazemi ${ }^{2}$, Kristopher Innanen ${ }^{3}$, Roman J. Shor ${ }^{4}$
}

\begin{abstract}
In this paper we consider the axial motion of a drill-string and the interaction of the drill-bit with the formation. We design an observer that estimates, in real time, the axial speed and force along the drill-string and at the drillbit using only topside measurements (force and velocity). More generally, our approach enables the design of robust observers for systems of Ordinary Differential Equation coupled with a first order Partial Differential Equation in their actuation path (a class of systems to which belongs the considered drillstring axial dynamics). However, such an observer requires the knowledge of different physical parameters among which the rock intrinsic energy which is a priori unknown. Thus, we combine our observer with an algorithm that provides a near real-rime estimation of the seismic velocities of rocks interacting with the drill-bit, using seismic-while-drilling. The efficiency of the proposed approach is shown through simulation results.
\end{abstract}

\section{INTRODUCTION}

Accessing subsurface deposits of hydrocarbons and other forms of energy, geothermal for example, requires both a detailed knowledge of the subsurface reservoirs as well as an efficient method for accessing them. Currently, our knowledge of the subsurface mainly comes from the use of seismic data, which contains information about geologic structures through reflections of energy across reflection boundaries, from the sparse well log and well core data in certain areas, and from the study of outcrops, where formations are exposed at the surface. In seismic data processing, the depth velocity model building is an integral part of migrating the reflected energy and tying the events to the well $\operatorname{logs}$ [14]. Direct velocity inversion of seismic data is another possibility [8]. However, depending on the complexity of the subsurface structure, the velocity model provided by seismic data processing may suffer from uncertainties. Seismic-while-drilling (SWD) is another tool that can provide the velocity of subsurface and can, in theory, reduce the uncertainties of depth velocity models. SWD method has existed for some time [21], but has been limited in use due to sensing and operational requirements [19]. By combining a field validated, real-time model of the axial dynamics of a drill-string, the sensing requirements may be reduced if bit-rock interaction is estimated using surface measurements. This paper introduces such a model and then presents the necessary observer construct to estimate both the drill-string dynamics as well as the seismic wave velocity of the rock at the bit. Our approach for the observer design relies on the backsteppping method.

\footnotetext{
${ }^{1}$ Jean Auriol is with Université Paris-Saclay, CNRS, CentraleSupélec, Laboratoire des Signaux et Systèmes, 91190, Gif-sur-Yvette, France. jean.auriolecentralesupelec.fr ${ }^{2}$ Nasser Kazemi is with University of Calgary, Department of Petroleum Engineering, Department of Geoscience, CREWES and Integrated Drilling Research Laboratory, Calgary, Canada ${ }^{3}$ Kristopher Innanen is with University of Calgary, Department of Geoscience, CREWES and Integrated Drilling Research Laboratory, Calgary, Canada ${ }^{4}$ Roman J. Shor is with University of Calgary, Department of Petroleum Engineering and Integrated Drilling Research Laboratory, Calgary, Canada
}

This paper presents the axial drill-string model in Section II, and then derives the general observer for ODE-PDE type systems in Section III. This construct is then applied to estimate the intrinsic energy of the rock in Section IV and we conclude with a simulation example in Section V.

\section{DRILL-STRING MODEL}

In this section we describe the mechanical dynamics of the drilling system.

\section{A. Distributed axial dynamics of the drill-string}

The drilling system we consider is composed of three parts: a top-drive, the drill-string and the drill-bit. We consider the case of a vertical well with a roller-cone drill-bit. The top-drive is typically a large geared AC motor which imparts rotary motion to the drill-string around its main axis. The drill-string is a segmented pipe which extend from the top-drive at surface to the drill-bit at the bottom of the hole and is composed of 10-25 meters long segments of jointed pipe. The drill-string may have a bottom hole assembly (BHA) consisting of heavier pipe at the bottom. Assuming elastic deformations, we can obtain the dynamics of interests using the equations of continuity and state. We denote $\xi(t, x)$ the axial displacement of the drill-string. It is a function of $(t, x)$ which is evolving in $\{(t, x) \mid 0<t<T, x \in[0, L]\}$ (where $L$ is the total length of the drill-string and $T$ is a positive time). Using the distributed model given in [12], [15], the axial motion satisfies the following wave Partial Differential Equation

$$
\frac{\partial^{2} \xi}{\partial t^{2}}(t, x)-c_{\xi}^{2} \frac{\partial^{2} \xi}{\partial x^{2}}(t, x)=-k_{\xi} \frac{\partial \xi}{\partial t}(t, x),
$$

where $c_{\xi}=\sqrt{\frac{E}{\rho}}, \rho$ is the pipe mass density, $E$ its Young's modulus, and $k_{\xi}$ is a damping coefficient representing the viscous shear stresses acting on the pipe. The axial force associated to $\xi$ can be found from the strain, given as the local relative compression:

$$
w(t, x)=A_{s}(x) E \frac{(\xi(t, x)-\xi(t, x+d x))}{d x},
$$

$A_{s}(x)$ being the cross-sectional area of the drill-string and may vary with length and $d x \rightarrow 0$ the infinitesimal axial position increment. We will however assume here that it is constant. The axial velocity satisfies

$$
v(t, x)=\frac{\partial \xi(t, x)}{\partial t} .
$$

Using (1), we have that the axial velocity and force satisfy the following set of PDEs

$$
\frac{\partial w(t, x)}{\partial t}+A_{s} E \frac{\partial v(t, x)}{\partial x}=0
$$




$$
\frac{\partial v(t, x)}{\partial t}+\frac{1}{A_{s} \rho} \frac{\partial w(t, x)}{\partial x}=-k_{\xi} v(t, x) .
$$

Below we introduce the two boundary conditions (top-drive actuation and downhole boundary condition).

The topside weight on the drill-string, $w(t, 0)$, corresponds to the system actuation. The downhole boundary condition at $x=L$ is obtained from a force balance on the lumped Bottom-Hole Assembly (BHA). These two boundary conditions will be discussed in the following.

\section{B. Topside boundary condition}

For the considered drilling system, the top-drive suspended over the drill floor by the traveling block and is connected to the drill-string at the top. The traveling block is connected by several drill lines with one attached to the deadline anchor and the other being spooled on a drum controlled by AC induction motors [10]. Thus, we can consider that the system actuation corresponds to the topside weight $w_{0}(t)=w(t, 0)$. Considering $w_{0}$ as an input, we have

$$
-E A_{s} \frac{\partial \xi(t, x)}{\partial x}=w_{0}(t) .
$$

\section{Downhole boundary condition: bit-rock interaction}

The lowermost part of the drilling system is usually composed of drill collars that (due to their added inertia) have an impact on the global dynamics. The change in characteristic line impedance due to the transition from pipes to collars causes reflections in the traveling wave [1]. Rigorously speaking, we have to write a new wave PDE (similar to (1) but using the BHA density and Young's modulus) for the BHA. Nevertheless, the effect of this PDE can be lumped into an ODE coupled with the drill-string [12] as the length of the BHA $(\approx 200 \mathrm{~m})$ is much smaller than the one of the drill-string $(\approx 2000 \mathrm{~m})$. Thus, the downhole boundary condition at $x=L$ can be obtained from a force balance on the lumped BHA. This yields

$$
M_{b} \frac{\partial v}{\partial t}(t, L)=-w_{b}(v(t, L), w(t, L))+w(t, L),
$$

where $M_{b}$ is the mass of the lumped BHA and $w_{b}(\cdot, \cdot)$ the force acting from the rock on the BHA through the drilling bit, known as the weight on bit (WOB). The WOB is related to the bit velocity by considering the combined depth of cut per revolution [10], [15], [23]. The combined depth of cut per revolution $d(t)$ verifies,

$$
d(t)=\frac{v(t, L)}{\omega_{\text {bit }}},
$$

where, for simplicity, the bit angular velocity $\omega_{\text {bit }}$ is assumed constant here. We have previously presented a fully validated torsional model to estimate $\omega_{\text {bit }}$ which may be integrated with the model presented here through a coupling at the drillbit, but this is left for future work [4]. The cutting force is finally expressed as

$$
w_{b}(v(t, L), w(t, L))=w_{f}+K d(t)=w_{f}+a \zeta \epsilon d(t),
$$

where $w_{f}$ is a friction weight independent of the bit velocity (and therefore constant) while $K=a \zeta \epsilon$, with $a$ being the bit radius, $\zeta$ a characterization of the cutting angle and $\epsilon$ the intrinsic specific energy of the rock [11], [23]. Thus, the boundary condition (6) rewrites as

$$
M_{b} \frac{\partial^{2} \xi}{\partial t^{2}}(t, L)=-\frac{a \zeta \epsilon}{\omega_{\mathrm{bit}}} \frac{\partial \xi(t, L)}{\partial t}-w_{f}-E A_{s} \frac{\partial \xi(t, L)}{\partial x} .
$$

\section{Derivation of Riemann invariants}

The Riemann invariants of a hyperbolic PDE are the states corresponding to a transformation of the system which has a diagonalized transport matrix, i.e. the system can be written as a series of transport equations only coupled through the source terms [18]. On the set $\{(t, x) \mid 0<t<T, \quad x \in$ $[0, L]\}$, we define the Riemann invariants as

$$
\begin{aligned}
u(t, x) & =\left(\frac{\partial}{\partial t} \xi(t, x)-c_{\xi} \frac{\partial}{\partial x} \xi(t, x)\right) \mathrm{e}^{\frac{k_{\xi}}{2 c_{\xi}} x}, \\
z(t, x) & =\left(\frac{\partial}{\partial t} \xi(t, x)+c_{\xi} \frac{\partial}{\partial x} \xi(t, x)\right) \mathrm{e}^{-\frac{k_{\xi}}{2 c_{\xi}} x} .
\end{aligned}
$$

The exponential term is used to remove some diagonal terms that appear when performing the change of variables. This transformation enables us to rewrite (1) as the PDE system

$$
\begin{gathered}
\frac{\partial}{\partial t} u(t, x)+c_{\xi} \frac{\partial}{\partial x} u(t, x)=-\frac{k_{\xi}}{2} \mathrm{e}^{\frac{k_{\xi}}{c_{\xi}} x} z(t, \xi), \\
\frac{\partial}{\partial t} z(t, x)-c_{\xi} \frac{\partial}{\partial x} z(t, x)=-\frac{k_{\xi}}{2} \mathrm{e}^{-\frac{k_{\xi}}{c_{\xi}} x} u(t, \xi) .
\end{gathered}
$$

The boundary condition (5) rewrites

$$
u(t, 0)=z(t, 0)+\frac{2 c_{\xi}}{E A_{s}} w_{0}(t),
$$

while the boundary condition (8) rewrites

$$
\begin{aligned}
& z(t, L)=-\mathrm{e}^{\frac{k_{\xi}}{c_{\xi}} L} u(t, L)+2 \mathrm{e}^{-\frac{k_{\xi}}{2 c_{\xi}} L} X(t), \\
& \dot{X}(t)=-\frac{a \zeta \epsilon}{M_{b} \omega_{\mathrm{bit}}} X(t)-\frac{w_{f}}{M_{b}} \\
& -\frac{E A_{s}}{2 c_{\xi} M_{b}}\left(z(t, L) \mathrm{e}^{\frac{k_{\xi}}{2 c_{\xi}} L}-u(t, L) \mathrm{e}^{-\frac{k_{\xi}}{2 c_{\xi}} L}\right) .
\end{aligned}
$$

Note that the parameter $\epsilon$ depends on the nature of the rock and may be unknown.

\section{E. Problem under consideration}

The objective of this paper is to design an observer that estimates, in real time, the axial velocity and force all along the drill-string using only topdrive measurements. More precisely, we assume that we measure $w(t, 0)$ and $v(t, 0)$. To design the observer we will use the Riemann coordinates and work on the system (11)-(15) considering that $u(t, 0)$ and $z(t, 0)$ are known. Although the considered system is stable, the proposed observer will have a faster convergence rate and will be combined with an estimation of $\epsilon$ in real time. The system (11)-(15) is actually a specific case of an interconnected ODE-PDE system. In the next section we design an observer for this general class of system.

\section{A GENERAL OBSERVER FOR INTERCONNECTED ODE-PDES SYSTEM}

Let us consider the following general system composed of an ODE coupled through its actuation path with a linear first-order hyperbolic system:

$$
\begin{aligned}
\partial_{t} u(t, x)+\lambda \partial_{x} u(t, x) & =\sigma^{+}(x) v(t, x), \\
\partial_{t} v(t, x)-\mu \partial_{x} v(t, x) & =\sigma^{-}(x) u(t, x), \\
\dot{X}(t) & =A X(t)+B u(t, L)+D,
\end{aligned}
$$

evolving in $\{(t, x)$ s.t. $t>0, x \in[0, L]\}$, with the boundary conditions

$$
u(t, 0)=q v(t, 0)+V(t),
$$




$$
v(t, L)=\rho u(t, L)+C X(t),
$$

where $X \in \mathbb{R}^{p}$ is the ODE state, and $u(t, x) \in \mathbb{R}, v(t, x) \in$ $\mathbb{R}$ are the PDE states. We assume that $-\mu<0<\lambda$. The constant real boundary coupling defined by $q$ (distal reflection) and $\rho \neq 0$ (proximal reflection) are constants. The in-domain coupling terms $\sigma^{+}$and $\sigma^{-}$are continuous functions. The initial conditions $\left(u_{0}, v_{0}\right)$ of the state $(u, v)$ are $L^{2}([0, L], \mathbb{R})$ functions. The actuation is denoted $V(t) \in$ $\mathbb{R}$. The matrices $A, B, C$ and $D$ are constant. The initial condition of the ODE (18) is denoted $X_{0}$. Such a system is well-posed [7]. It is straightforward to rewrite the Riemann axial motion equations (11)-(15) in the form (16)-(17). We consider that we have collocated measurements, i.e., we measure $y(t)=v(t, 0)$. In the rest of this section, we design an observer for the system (16)-(19) that will provide an estimation of the states $u(t, x), v(t, x), X(t)$ in real time. In what follows we denote $\tau$ as the sum of the transport velocities in both directions: $\tau=\frac{1}{\lambda}+\frac{1}{\mu}$. We will consider the norm $\|\cdot\|$ defined by

$$
\|(u, v, X)\|=\|u\|_{L^{2}}+\|v\|_{L^{2}}+\|X\|_{\mathbb{R}^{p}} .
$$

We make the following assumptions

Assumption 1: The boundary couplings verify $|\rho q|<1$.

Assumption 2: The pair (A,C) is observable, that is there exists a matrix $L$ such that $A-L C$ is Hurwitz.

This second assumption is necessary to estimate the ODE state $X(t)$, while the first one guarantees the possibility to design a delay-robust observer (see [3] for details). These assumptions are satisfied for the system (11)-(15).

\section{A. Observer equations}

Inspired by [26], the observer equations read as follows

$$
\begin{aligned}
\partial_{t} \hat{u}+\lambda \partial_{x} \hat{u} & =\sigma^{+}(x) \hat{v}-p_{1}(x)(v(t, 0)-\hat{v}(t, 0)), \\
\partial_{t} \hat{v}-\mu \partial_{x} \hat{v} & =\sigma^{-}(x) \hat{u}-p_{2}(x)(v(t, 0)-\hat{v}(t, 0)), \\
\dot{\hat{X}}(t) & =A \hat{X}(t)+B \hat{u}(t, L)+D \\
& -\mathcal{P}_{0}(v(t, 0)-\hat{v}(t, 0)),
\end{aligned}
$$

with the boundary conditions

$$
\begin{aligned}
& \hat{u}(t, 0)=q \hat{v}(t, 0)+V(t), \\
& \hat{v}(t, L)=\rho \hat{u}(t, L)+C \hat{X}(t),
\end{aligned}
$$

where $(\hat{u}, \hat{v}, \hat{X})$ is the estimate of the state $(u, v, X)$, the terms, $p_{1}, p_{2}$ are output injection gains ( $L^{\infty}$ functions defined on $\left.([0, L])^{2}\right)$ that have to be designed. We have $\mathcal{P}_{0}$ that is a linear operator acting on $v(t, 0)-\hat{v}(t, 0)$. More precisely, $\mathcal{P}_{0}$ is an operator defined on $D$, where we have denoted $D=L^{2}\left(\left[-\tau-\frac{1}{\lambda}, 0\right] ; \mathbb{R}\right)$ the Banach space of $L^{2}$ functions mapping the interval $\left[-\tau-\frac{1}{\lambda}, 0\right]$ into $\mathbb{R}$. For a function $z:\left[-\tau-\frac{1}{\lambda}, \infty\right) \rightarrow \mathbb{R}$, we define its partial trajectory $z_{[t]} \in D$ by $z_{[t]}(\theta)=z(t+\theta), \quad-\tau-\frac{1}{\lambda} \leq \theta \leq 0$. The initial conditions of this observer are $L^{2}$ functions. Defining the error estimates $\tilde{u}=u-\hat{u}, \tilde{v}=v-\hat{v}$ and $\tilde{X}=X-\hat{X}$, the error system is obtained by subtracting the observer equations in (20)-(23) from (16)-(19),

$$
\begin{aligned}
& \partial_{t} \tilde{u}+\lambda \partial_{x} \tilde{u}=\sigma^{+}(x) \tilde{v}+p_{1}(x) \tilde{v}(t, 0), \\
& \partial_{t} \tilde{v}-\mu \partial_{x} \tilde{v}=\sigma^{-}(x) \tilde{u}+p_{2}(x) \tilde{v}(t, 0), \\
& \dot{\tilde{X}}(t)=A \tilde{X}(t)+B \tilde{u}(t, L)+\mathcal{P}_{0}(\tilde{v}(t, 0)),
\end{aligned}
$$

with the boundary conditions

$$
\begin{aligned}
\tilde{u}(t, 0) & =q \tilde{v}(t, 0), \\
\tilde{v}(t, L) & =\rho \tilde{u}(t, L)+C \tilde{X}(t) .
\end{aligned}
$$

We now need to find the output injection gains $p_{1}, p_{2}$ and the operator $\mathcal{P}_{0}$ such that the error system decays to zero (which implies the convergence of the estimated state to the real one).

\section{B. Backstepping transformation}

In this section, we use a backstepping transformation [17] to map the system (24)-(26) into a target system for which the analysis is simpler. The original system and the corresponding target system have equivalent properties [17]. More precisely, inspired by [9], we consider the following transformation

$$
\begin{aligned}
& \tilde{u}=\tilde{\alpha}-\int_{0}^{x} K^{\alpha \alpha}(x, \xi) \tilde{\alpha}(\xi)+K^{\alpha \beta}(x, \xi) \tilde{\beta}(\xi) d \xi \\
& \tilde{v}=\tilde{\beta}-\int_{0}^{x} K^{\beta \alpha}(x, \xi) \tilde{\alpha}(\xi)+K^{\beta \beta}(x, \xi) \tilde{\beta}(\xi) d \xi \\
& \tilde{Y}=\tilde{X}-\int_{0}^{L} L^{\alpha}(\xi) \tilde{\alpha}(\xi)+L^{\beta}(\xi) \tilde{\beta}(\xi) d \xi
\end{aligned}
$$

where the different kernels $K^{\alpha \alpha}, K^{\alpha \beta}, K^{\beta \alpha}, K^{\beta \beta}$ are $L^{\infty}$ functions defined on $\mathcal{T}=\left\{(x, \xi) \in[0, L]^{2} \mid x \geq\right.$ $\xi\}$, while the kernels $L^{\alpha}$ and $L^{\beta}$ are defined on $[0,1]$. The transformation (28)-(30) is invertible as it corresponds to an invertible Volterra transformation [28]. The kernels $K^{\alpha \alpha}, K^{\alpha \beta}, K^{\beta \alpha}, K^{\beta \beta}, L^{\alpha}, L^{\beta}$ satisfy the following set of PDEs and ODEs

$$
\begin{aligned}
& \lambda \partial_{x} K^{\alpha \alpha}+\lambda \partial_{\xi} K^{\alpha \alpha}=\sigma^{+}(x) K^{\beta \alpha}, \\
& \lambda \partial_{x} K^{\alpha \beta}-\mu \partial_{\xi} K^{\alpha \beta}=\sigma^{+}(x) K^{\beta \beta}, \\
& \mu \partial_{x} K^{\beta \alpha}-\lambda \partial_{\xi} K^{\beta \alpha}=-\sigma^{-}(x) K^{\alpha \alpha}, \\
& \mu \partial_{x} K^{\beta \beta}+\mu \partial_{\xi} K^{\beta \beta}=-\sigma^{-}(x) K^{\alpha \beta},
\end{aligned}
$$

with the ODEs

$$
\begin{aligned}
& \lambda \partial_{\xi} L^{\alpha}(\xi)=-B K^{\alpha \alpha}(L, \xi)+A L^{\alpha}(\xi), \\
& \mu \partial_{\xi} L^{\beta}(\xi)=B K^{\alpha \beta}(L, \xi)-A L^{\beta}(\xi),
\end{aligned}
$$

and the boundary conditions

$$
\begin{aligned}
& K^{\alpha \beta}(x, x)=-\frac{\sigma^{+}(x)}{\lambda+\mu}, K^{\beta \alpha}(x, x)=\frac{\sigma^{-}(x)}{\lambda+\mu}, \\
& K^{\beta \beta}(L, \xi)=\rho K^{\alpha \beta}(L, \xi)-C L^{\beta}(\xi), \\
& K^{\alpha \alpha}(L, \xi)=\frac{1}{\rho} K^{\beta \alpha}(L, \xi)+\frac{C}{\rho} L^{\alpha}(\xi), \\
& L^{\beta}(L)=0, \quad L^{\alpha}(L)=0 .
\end{aligned}
$$

The well-posedness of the system (31)-(40) is assessed by the following lemma

Lemma 1: Consider system (31)-(40). There exists a unique solution $K^{\alpha \alpha}, K^{\beta \alpha}, K^{\alpha \beta}, K^{\beta \beta}$ in $L^{\infty}(\mathcal{T})$ and two $\mathcal{C}^{1}$ functions $L^{\alpha}$ and $L^{\beta}$.

Proof: The proof can be found in [5, Lemma 1] which is adjusted from the one given in [13].

We now set the observer gains as

$$
\begin{aligned}
& p_{1}(x)=\mu K^{\alpha \beta}(x, 0)-\lambda q K^{\alpha \alpha}(x, 0), \\
& p_{2}(x)=\mu K^{\beta \beta}(x, 0)-\lambda q K^{\beta \alpha}(x, 0) .
\end{aligned}
$$


Differentiating (28)-(29) with respect to time and space, and using the fact that $\tilde{\beta}(t, 0)=\tilde{v}(t, 0)$, we can show that it maps the system (24)-(26) to the target system

$$
\begin{aligned}
& \partial_{t} \tilde{\alpha}(t, x)+\lambda \partial_{x} \tilde{\alpha}(t, x)=0, \\
& \partial_{t} \tilde{\beta}(t, x)-\mu \partial_{x} \tilde{\beta}(t, x)=0, \\
& \dot{\tilde{Y}}(t)=A \tilde{Y}(t)+\tilde{\mathcal{P}}_{0}(\tilde{\beta}(t, 0)),
\end{aligned}
$$

with the boundary conditions

$$
\begin{aligned}
& \tilde{\alpha}(t, 0)=q \tilde{\beta}(t, 0), \\
& \tilde{\beta}(t, L)=\rho \tilde{\alpha}(t, L)+C \tilde{Y}(t),
\end{aligned}
$$

where $\tilde{\mathcal{P}}_{0}$ is the linear operator defined on $D$ by

$$
\begin{aligned}
\tilde{\mathcal{P}}_{0}(\tilde{\beta}(t, 0))= & \mathcal{P}_{0}(\tilde{\beta}(t, 0))+B q \tilde{\beta}\left(t-\frac{1}{\lambda}, 0\right) \\
& +\left(\lambda q L^{\alpha}(0)-\mu L^{\beta}(0)\right) \tilde{\beta}(t, 0) .
\end{aligned}
$$

\section{Design of the linear operator $\tilde{\mathcal{P}}_{0}$}

Equations (43) and (44) are transport equations. Consequently, for $t \geq \tau$, using the boundary conditions (46), we obtain

$$
\tilde{\beta}(t, 0)=\rho q \tilde{\beta}(t-\tau, 0)+C \tilde{Y}\left(t-\frac{1}{\mu}\right) .
$$

Let us now define $\tilde{\mathcal{P}}_{0}$ as the linear operator defined on $D$ by

$$
\begin{aligned}
\tilde{\mathcal{P}}_{0}: D & \rightarrow \mathbb{R} \\
z_{[t]} & \mapsto-\mathrm{e}^{\frac{A}{\mu}} L\left(\tilde{\mathcal{P}}_{1}\left(z_{[t]}(0)\right)-\rho q \tilde{\mathcal{P}}_{1}\left(z_{[t]}(-\tau)\right)\right),
\end{aligned}
$$

where $L$ is a matrix such that $A-L C$ is Hurwitz (see Assumption 2) and where the linear operator $\tilde{\mathcal{P}}_{1}$ defined on $D$ still has to be designed. We have the following Theorem

Theorem 1: Let us denote $\overline{\mathcal{P}}_{1}(t)=\tilde{\mathcal{P}}_{1}(\tilde{\beta}(t, 0))$ the function defined by

$$
\overline{\mathcal{P}}_{1}(t)=\tilde{\beta}(t, 0)-C \int_{t-\frac{1}{\mu}}^{t} \mathrm{e}^{A(t-\nu)} L \overline{\mathcal{P}}_{1}(\nu) d \nu,
$$

Then, the state $Y(t)$ solution of (45) exponentially converges to 0 .

Proof: Let us consider the operator $\overline{\mathcal{P}}_{1}$ defined by (50). Let us denote $P_{0}(t)=\overline{\mathcal{P}}_{1}(t)-\rho q \overline{\mathcal{P}}_{1}(t-\tau)$. With this operator equation (45) rewrites

$$
\begin{aligned}
\dot{\tilde{Y}} & =A \tilde{Y}-\mathrm{e}^{\frac{A}{\mu}} L P_{0}(t), \\
P_{0}(t)= & \tilde{\beta}(t, 0)-\rho q \tilde{\beta}(t-\tau, 0) \\
& -C \int_{t-\frac{1}{\mu}}^{t} \mathrm{e}^{A(t-\nu)} L P_{0}(\nu) d \nu .
\end{aligned}
$$

Using (48), equation (52) rewrites

$$
P_{0}(t)=C \tilde{Y}\left(t-\frac{1}{\mu}\right)-C \int_{t-\frac{1}{\mu}}^{t} \mathrm{e}^{A(t-\nu)} L P_{0}(\nu) d \nu
$$

This corresponds to the error system for the observer with delayed measurements given in [16, Remark 3]. We can then conclude $\tilde{Y}$ exponentially converges to zero.

Remark 1: As explained in [16], the proposed operator observer (50) is not the only possible design. Reducedorder observers have been designed in [27] for instance. The benefit of employing such a full-order observer is the reduced sensitivity to measurement noise.

With this definition for the operator $\mathcal{P}_{0}$, equation (26) can be rewritten as

$$
\begin{aligned}
& \dot{\hat{X}}(t)=A \hat{X}(t)+B \hat{u}(t, L)+\left(\lambda q L^{\alpha}(0)-\rho L^{\beta}(0)\right) \tilde{v}(t, 0) \\
& +\mathrm{e}^{\frac{A}{\mu}} L(P(t)-\rho q P(t-\tau))+B q \tilde{v}\left(t-\frac{1}{\lambda}, 0\right) \\
& P(t)=\tilde{v}(t, 0)-C \int_{t-\frac{1}{\mu}}^{t} \mathrm{e}^{A(t-\nu)} L P(\nu) d \nu
\end{aligned}
$$

Theorem 2: The observer (20)-(23), and (54), where $p_{1}$ and $p_{2}$ are defined by (41) and (42) exponentially converges to the real solution of (16)-(19)

Proof: Due to the analysis done above and due to Theorem 1, we have that $Y(t)$ exponentially converges to 0 . Since $|\rho q|<1$ (which means that the complex operator $s \mapsto 1-\rho q \mathrm{e}^{-\tau s}$ has all its roots on the complex left half plane), we have from (48) that the state $\beta(t, 0)$ converges to zero, which in turn implies from (44) that $\beta(t, \cdot)$ converges exponentially to zero. This implies from (43) and the boundary condition (46) the convergence of $\alpha(t, \cdot)$ to zero. Using the invertibility of the transformation (28)-(30), we have the exponential convergence of the state $(\tilde{u}, \tilde{v}, \tilde{X})$ to zero. This concludes the proof.

Remark 2: Note that due to the fact that we do not cancel the reflection term $\rho$ in the observer (20)-(23) guarantees some robustness margins (see [5] for details). However, the convergence rate can be increased by cancelling a part of this reflection.

This observer can now be applied on the original system (11)-(15) to determine the axial force and velocity. However, in equation (15), the parameter $\epsilon$ depends on the nature of the drilled rock. This coefficient is not known a priori. In the next section we describe an algorithm used to estimate it.

\section{ESTIMATION OF THE SPECIFIC INTRINSIC ENERGY $\epsilon$}

The specific intrinsic energy of rocks that the system is drilling into is one of the main input parameters to the observer. In this section, we show how to estimate this value for different rocks. The intrinsic specific energy refers to the amount of energy that is required to cut a unit volume of rock. This value is dependent on cutter geometry, depth of cut, and rock. However, similar to scratch test studies in rock mechanics, we assume that the specific intrinsic energy is a constant quantity characterizing a particular combination of cutter geometry and rock. Richard and Dagrain [24], by using different rock types, study the relationship between the $\epsilon$ and the Uniaxial Compressive Strength (UCS). They show that by expressing the $\epsilon$ as a stress rather than energy, the $\epsilon$ is correlated with UCS

$$
\epsilon(M P a) \approx U C S(M P a)
$$

Also, several recent works aim at relating the rock strength to the seismic velocity of primary wave [25]. For example, by comprehensive analysis of different rock types, ranging from sedimentary to metamorphic, Sharma and Singh [25] show that the seismic velocity of primary wave and UCS are linearly correlated and the relationship is as follows

$$
U C S=0.0642 V_{p}-117.99,
$$


where $V_{p}(\mathrm{~m} / \mathrm{s})$ is the seismic velocity of primary wave. This relationship is derived based on linear regression and a strong correlation $R^{2}=0.9022$ is reported. By plugging equation (57) into equation (56), we get

$$
\epsilon \approx 0.0642 V_{p}-117.99 \text {. }
$$

Hence, by knowing the seismic velocity of the rock that the system is drilling into the estimation of $\epsilon$ is possible. Next, we show how to estimate the primary wave velocity of rocks while drilling.

Seismic while drilling (SWD) measurement records the radiated elastic energy from the drillbit-rock interaction that is traveled through the earth's structure. SWD measurements can provide the drill-bit position through checkshots, sonic calibrations through reflectivity characterization, prediction ahead of the bit through reverse VSP, and subsurface structure around and ahead of the drill-bit through multi-offset VSP. Moreover, variations in the amplitude of the direct arrivals of P-waves and S-waves in the processed SWD measurements, after removing the source signature, reflect the changes in the rock properties near the drill-bit. For example, changes in the energy of the direct arrivals of P-waves and $\mathrm{S}$-waves can imply that the bit is turning right and that the trajectory of well is modified. The direct arrivals can be used in a relative sense to infer the relative changes in the rock properties such as their seismic velocities or unconstrained rock strengths. However, Rector and Hardage [22] show that the radiation patterns of the direct arrival depend on the primary wave velocity of the rock interacting with the drillbit, and, in our recent work [6], we develop an inversion algorithm to solve for such velocity. The radiation pattern for the primary wave is as follows

$$
U_{r}(r, \phi, t)=\frac{A_{1} \cos (\phi)}{\rho_{f} V_{p}^{2} r} \hat{w}\left(t-\frac{r}{V_{p}}, L\right),
$$

where $r$ is the distance from drill-bit to the receiver, $\phi$ is the opening angle between the drill-bit and receiver, measured relative to the drill-string, $t$ is time, $L$ is the length of drillstring, $\rho_{f}$ is the density of the interacting rock with the drillbit, $\hat{w}=w \odot w$ is the auto-correlation of drill-bit source signature, $A_{1}$ is a constant, and $U_{r}$ is the radial component of the primary wave radiation pattern. In most rocks we have $\rho_{f}=1.74 V_{p}^{0.25}$, hence equation (58) simplifies to

$$
U_{r}(r, \phi, t)=\frac{A_{1} \cos (\phi)}{1.74 V_{p}^{2.25} r} \hat{w}\left(t-\frac{r}{V_{p}}, L\right) .
$$

By matching the direct arrival of the primary wave in the source compensated SWD data to the radiation pattern in equation (60), we can estimate the velocity of rock interacting with the drill-bit. However, there are two unknowns in equation (60), i.e., $V_{p}$ and $w$. Hence, first, we need to estimate the drill-bit source signature. To do so, in our recent work [6], we show that by distributed modeling of the dynamics of the drill-string, it is possible to express the drill-bit source signature as a function of top-drive force and velocity measurements

$$
\begin{aligned}
w(t, L)= & \frac{E A}{2 c_{\xi}}\left(\mathrm{e}^{-\frac{k_{\xi} L}{2 c_{\xi}}} v\left(t-\frac{L}{c_{\xi}}, 0\right)-\mathrm{e}^{\frac{k_{\xi} L}{2 c_{\xi}}} v\left(t+\frac{L}{c_{\xi}}, 0\right)\right) \\
& +\frac{1}{2}\left(\mathrm{e}^{-\frac{k_{\xi} L}{2 c_{\xi}}} w\left(t-\frac{L}{c_{\xi}}, 0\right)+\mathrm{e}^{\frac{k_{\xi} L}{2 c_{\xi}}} w\left(t+\frac{L}{c_{\xi}}, 0\right)\right)
\end{aligned}
$$

$$
\begin{aligned}
& +\int_{-\frac{L}{c_{\xi}}}^{\frac{L}{c_{\xi}}}\left(f_{u}(s)+f_{z}(s)\right) v(t-s, 0) d s \\
& +\int_{-\frac{L}{c_{\xi}}}^{\frac{L}{c_{\xi}}} \frac{c_{\xi}}{E A}\left(f_{u}(s)-f_{z}(s)\right) w(t-s, 0) d s .
\end{aligned}
$$

where the functions $f_{u}, f_{z}$ depend on the parameters of the problem. Now that we have the estimate of the drill-bit source signature $w$, the only unknown in equation (60) is the primary velocity of rock, i.e., $V_{p}$. The cross-correlation of the estimated source signature by using equation (61) with the SWD data gives the primary direct arrival. By least-squares matching of the primary direct arrival in the source compensated SWD data, $U_{r}^{o b s}(r, \phi, t)$, with the radiation pattern represented in equation (60) and after plugging the autocorrelation of the estimated source signature, we estimate the primary wave velocity of the rock. Finally, after estimating the primary wave velocity of rock, we estimate $\epsilon$ using (58).

\section{Simulation Results}

In this section we illustrate our results with simulation of a simple field scenario. A vertical wellbore is placed into a layered homogeneous geology model simulating a simple deep conventional reservoir. We consider a drill-string whose axial motion satisfies the PDE given by equation (1) subject to the boundary conditions given in (5)-(8). Table I shows the numerical values of parameters for the drillstring system. The drill-bit is interacting with unconsolidated sands $\left(\epsilon=11 \mathrm{Jcm}^{-3}\right)$. The PDE system is simulated using a classical finite volume method based on a Godunov scheme [18]. The control input $w_{0}$ is chosen as a sinusoidal function, thus allowing this to simulate either roller cone or PDC bits. A numerical solution to equations (31)-(40) can be reached with the method of characteristics and a fixed point algorithm (see [2]). We want to estimate in real time the state $(w, v, v(t, L))$ (where $w$ is the force, $v$ the velocity and $v(\cdot, L)$ the state at the bit) using the observer given in Section III combined with an estimation of $\epsilon$. The predictor given in (54) is adjusted from the one presented in [20]. Figure 1 pictures the $\|\cdot\|$-norm of the error state $(\tilde{w}, \tilde{v}, \tilde{v}(\cdot, L))$, difference between the real state and the estimated state $(\hat{w}, \hat{v}, \hat{v}(t, L))$ for three different situations. Note that the force has been expressed in MN. In the first case, we assume that $\epsilon$ is perfectly known. This results to a convergence of the error to zero. Then, we consider that $\epsilon$ known but set to a wrong value (here $\epsilon$ is set to $20 \mathrm{Jcm}^{-3}$ instead of $11 \mathrm{Jcm}^{-3}$ ). This results in an important error in the estimation. Finally, using the algorithm described in Section IV, we update the estimation of $\epsilon$ in real time. The function $U_{r}^{o b s}$ is obtained using the simulated weight-onbit and the (known) value of $V_{p}$, A white Gaussian noise with signal-to-noise ratio (SNR) equal to 10 (the SNR is the ration between the mean-square amplitude of the noise-free signal and the variance of the noise). By using the top-drive force and velocity measurements, which are given by the simulation of the PDE, we estimate the weight-on-bit (see equation (61)). As it can be seen in Figure 1, this results to a good estimation of the state $(w, v, v(t, L))$.

\section{CONCLUDING REMARKS}

In this paper, we presented the model for the axial dynamics of a drill-string which included the topside boundary condition and the bit-rock interaction boundary condition. 


\begin{tabular}{|c|c||c|c|}
\hline Param. & Value & Param. & Value \\
\hline \hline$E$ & $2 \times 10^{11} \mathrm{~Pa}$ & $\rho$ & $8000 \mathrm{~kg} \cdot \mathrm{m}^{-3}$ \\
$c_{\xi}$ & $5000 \mathrm{~m} . \mathrm{s}^{-1}$ & $k_{\xi}$ & $0.23 \mathrm{~s}^{-1}$ \\
$A$ & $3.5 \times 10^{-3} \mathrm{~m}^{2}$ & $L$ & $2000 \mathrm{~m}$ \\
$M_{b}$ & $30000 \mathrm{~kg}$ & $\omega_{b i t}$ & $30 \mathrm{~ms}^{-1}$ \\
$a$ & $0.1 \mathrm{~m}$ & $\zeta$ & 0.6 \\
$w_{f}$ & $71280 \mathrm{~N}$ & $\phi$ & $0.1745 \mathrm{rad}$ \\
\hline
\end{tabular}

TABLE I

NUMERICAL VALUES OF THE PARAMETERS

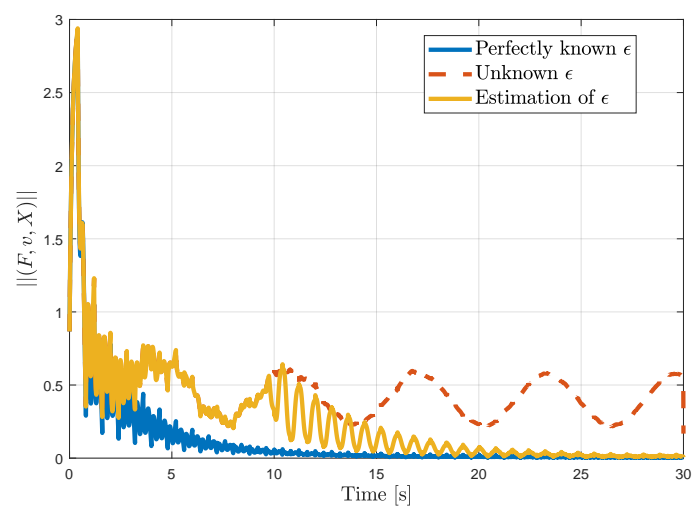

Fig. 1. Time evolution of the error-state using the observer given in Section III for a perfectly known $\epsilon$ (Case a)), a wrong value of $\epsilon$ (Case b)), estimating $\epsilon$ using the algorithm given in Section IV (Case c)).

We then derived an observer of the coupled ODE-PDE system and showed exponential convergence. Finally, we applied this construct to estimate, in real-time, the intrinsic rock strength of the rock based on the bit-rock interaction law. In simulation, we showed rapid convergence to the true model parameter, even in the case where the original guess of the parameter was incorrect. This presents an important advance, first in parameter estimation in ODE-PDE coupled systems and secondly for applications of seismic-whiledrilling methodology where rock properties may be estimated using solely surface data. Future work will combine this axial model with a similar, also validated, torsional model, to account for fully coupled axial-torsional dynamics.

\section{ACKNOWLEDGMENTS}

This work was supported by the University of Calgary's Canada First Research Excellence Fund Program, the Global Research Initiative in Sustainable Low Carbon Unconventional Resources.

\section{REFERENCES}

[1] U. J. F. Aarsnes and O. M. Aamo. Linear stability analysis of selfexcited vibrations in drilling using an infinite dimensional model. Journal of Sound and Vibration, 360:239-259, jan 2016.

[2] J. Auriol. Robust design of backstepping controllers for systems of linear hyperbolic PDEs. PhD thesis, PSL Research University, 2018.

[3] J. Auriol, U. J. F. Aarsnes, P. Martin, and F. Di Meglio. Delay-robust control design for heterodirectional linear coupled hyperbolic PDEs. IEEE Transactions on Automatic Control, 2018.

[4] J. Auriol, U. J. F. Aarsnes, and R. Shor. Self-tuning torsional drilling model for real-time applications. In American Control Conference (to appear), 2020.

[5] J. Auriol, F. Bribiesca Argomedo, D. Bou Saba, M. Di Loreto, and F. Di Meglio. Delay-robust stabilization of a hyperbolic PDE-ODE system. Automatica, 95:494-502, 2018.

[6] J. Auriol, N. Kazemi, R. J. Shor, K. A.. Innanen, and I. D. Gates. A sensing and computational framework for estimating the seismic velocities of rocks interacting with the drill-bit. IEEE Transactions on geoscience and remote sensing, pages 1-12, 2019.
[7] G. Bastin and J.-M. Coron. Stability and boundary stabilization of 1-d hyperbolic systems. Springer, 2016

[8] H. Ben-Hadj-Ali, S. Operto, and J. Virieux. Velocity model building by $3 \mathrm{~d}$ frequency-domain, full-waveform inversion of wide-aperture seismic data3d full-waveform tomography. Geophysics, 73(5):VE101VE117, 2008.

[9] D. Bou Saba, F. Bribiesca-Argomedo, M. Di Loreto, and D. Eberard. Strictly proper control design for the stabilization of $2 \times 2$ linear hyperbolic ODE-PDE-ODE systems. In 2019 IEEE 58th Annual Conference on Decision and Control (CDC), volume 2018-Janua, pages 2498-2503. IEEE, dec 2019.

[10] E. Cayeux. On the Importance of Boundary Conditions for Real-Time Transient Drill-String Mechanical Estimations. In IADC/SPE Drilling Conference and Exhibition. Society of Petroleum Engineers, mar 2018

[11] E. Detournay and P. Defourny. A phenomenological model for the drilling action of drag bits. International Journal of Rock Mechanics and Mining Sciences \& Geomechanics Abstracts, 29(1):13-23, 1992.

[12] F. Di Meglio and Ulf J. F. Aarsnes. A distributed parameter systems view of control problems in drilling. IFAC-PapersOnLine, 48(6):272$278,2015$.

[13] F. Di Meglio, F.B. Argomedo, L. Hu, and M. Krstic. Stabilization of coupled linear heterodirectional hyperbolic pde-ode systems. Automatica, 87:281-289, 2018.

[14] C. H. Dix. Seismic velocities from surface measurements. Geophysics, 20(1):68-86, 1955

[15] C. Germay, N. van de Wouw, H. Nijmeijer, and R. Sepulchre. Nonlinear Drillstring Dynamics Analysis. SIAM Journal on Applied Dynamical Systems, 8(2):527-553, jan 2009.

[16] M. Krstic and A. Smyshlyaev. Backstepping boundary control for first-order hyperbolic pdes and application to systems with actuator and sensor delays. Systems \& Control Letters, 57(9):750-758, 2008.

[17] M. Krstic and A. Smyshlyaev. Boundary Control of PDEs: A Course on Backstepping Designs. SIAM, 2008.

[18] R. J. LeVeque. Finite volume methods for hyperbolic problems. Cambridge university press, 2002.

[19] W. McDonald, G. Pittard, and F. Reuter. Seismic energy source for use during wellbore drilling, December 2 2004. US Patent App. $10 / 775,455$.

[20] S. Mondié and W. Michiels. Finite spectrum assignment of unstable time-delay systems with a safe implementation. IEEE Transactions on Automatic Control, 48(12):2207-2212, 2003.

[21] FB Poletto and Francesco Miranda. Seismic while drilling: Fundamentals of drill-bit seismic for exploration, volume 35. Elsevier, 2004.

[22] J. Rector III and B. Hardage. Radiation pattern and seismic waves generated by a working roller-cone drill bit. Geophysics, 57(10):13191333, 1992.

[23] T. Richard, C. Germay, and E. Detournay. A simplified model to explore the root cause of stick-slip vibrations in drilling systems with drag bits. Journal of Sound and Vibration, 305(3):432-456, aug 2007.

[24] Thomas Richard, Fabrice Dagrain, Edmond Poyol, and Emmanuel Detournay. Rock strength determination from scratch tests. Engineering Geology, 147:91-100, 2012.

[25] P. Sharma and T. Singh. A correlation between p-wave velocity, impact strength index, slake durability index and uniaxial compressive strength. Bulletin of Engineering Geology and the Environment, 67(1):17-22, 2008.

[26] R. Vazquez, J.-M. Coron, M. Krstic, and G. Bastin. Collocated outputfeedback stabilization of a $2 \times 2$ quasilinear hyperbolic system using backstepping. In 2012 American Control Conference (ACC), pages 2202-2207. IEEE, jun 2012.

[27] K. Watanabe and M. Ito. An observer for linear feedback control laws of multivariable systems with multiple delays in controls and outputs. Systems \& control letters, 1(1):54-59, 1981.

[28] K. Yoshida. Lectures on differential and integral equations, volume 10. Interscience Publishers, 1960. 